suffered a GD/GDV episode were fed from a height compared with 27 (53\%) unaffected dogs ( $p=0.032$ ). Significantly, a larger proportion of the dogs which suffered from GD/GDV suffered from borborygmi ( $p=0.037$ ). Seven Greyhound owners reported their dogs undergoing previous abdominal surgery, 6 of these went on to develop GD/GDV ( $p=0.0001)$. Six $(38 \%)$ Greyhounds suffering a GD/GDV had a relative which suffered from the condition compared with $5(24 \%)$ dogs which did not suffer bloat $(p=$ 0.026).Twelve (86\%) dogs undergoing surgery for the GD/ GDV survived and 9 (75\%) were subsequently discharged.

\section{STATEMENT (CONCLUSIONS)}

This study indicates that feeding from a height, presence of borborygmi, having an affected relative and previous abdominal surgery could be important factors influencing the development of GD/GDV in Greyhounds. This information may be valuable for Greyhound owners attempting to minimise the chances of their dogs developing GD/GDV.

\section{Effect of dog}

ownership experience

on the uptake of

preventative health

care practices

reported by puppy

owners

\section{Emma Buckland ${ }^{1}$, Rosa Da Costa ${ }^{1}$, Rachel Casey ${ }^{1}$, Michelle Lord ${ }^{1}$, Rachel Kinsman ${ }^{1}$, Joshua Woodward ${ }^{1}$, Séverine Tasker ${ }^{2,3}$, Toby Knowles ${ }^{2}$, Jane Murray ${ }^{1}$}

\footnotetext{
1 Dogs Trust, London, United Kingdom

2 University of Bristol, Bristol, United Kingdom

3 Linnaeus Group, Shirley, United Kingdom
}

\section{OBJECTIVES}

To summarise preventative health care practices reported by owners of puppies aged $\leq 16$ weeks, and to determine whether previous dog ownership affected engagement in preventative health care.

\section{METHODS}

Owners who acquired a single puppy at $\leq 11.5$ weeks of age and lived in the UK or Republic of Ireland were recruited onto a longitudinal cohort study. Online questionnaires were completed at study registration and when puppies were aged 16 weeks. Chi-squared tests were used to examine associations between previous dog ownership and the use of flea and worm treatment/ preventative products, vaccinations and pet insurance, on puppies at or before 16 weeks of age.

\section{RESULTS}

Of 1,288 owners, $1,118(87 \%)$ and $1,230(95 \%)$ reported that by 16 weeks of age their puppies had been administered at least one flea product and at least one worming product, respectively; $1,259(98 \%)$ of puppies had been vaccinated at least once and 1,066 (83\%) were insured by 16 weeks.

First-time puppy owners were more likely than owners with previous dog ownership experience to use a flea product (OR: 2.63 ; 95\% Cl: $1.66-4.16$ : $\mathrm{P}<0.001$ ) and have pet insurance (OR: 1.92; $95 \% \mathrm{Cl}: 1.33-2.79$ : $\mathrm{P}<0.001$ ) by 16 weeks of age, respectively. No differences were found for the use of worming products $(P=0.3)$ nor vaccinations by 16 weeks of age $(P=0.2)$.

\section{STATEMENT (CONCLUSIONS)}

Flea product use and pet insurance acquisition were less commonly reported than worming product use and vaccination amongst our cohort subjects. Factors, including previous dog ownership, may influence uptake of preventative health care, suggesting advice could be tailored to different audiences.

\title{
Retrospective analysis of Canine Bronchoalveolar Lavage submissions to referral veterinary laboratories in the UK
}

\section{Guy Davies ${ }^{1,2}$, Rand Wilson', Peter Graham ${ }^{1}$}

1 School of Veterinary Medicine and Science University of Nottingham, Nottingham,

United Kingdom

2 Finn Pathologists, Norfolk, United Kingdom

\section{OBJECTIVES}

The aim of this study was to investigate the prevalence of bacterial respiratory disease in dogs using the results of canine bronchoalveolar lavage (BAL) submissions. 


\section{Oral presentations}

\section{METHODS}

A retrospective epidemiological study was performed on the results of canine BAL submissions to three referral veterinary laboratories between July 2013 and July 2018. Data on signalment, microbial culture, and cytological findings were collected. Cases were excluded if insufficient information was available. Data was collated using Microsoft Excel and descriptive statistics were performed using GraphPad Prism.

\section{RESULTS}

2501 cases were collected from a 5 year period. Cases ranged in age from 1 to 17 years with a mean age of 7.87 years. $1735(69 \%)$ of those cases had microbial culture performed, with 767 yielding a positive culture. 405 cases
(16.2\%) had a cytological diagnosis of neutrophilic inflammation with sepsis, 237 of which had a concurrent positive microbial culture result. 1100 cases had findings of neutrophilic inflammation with no overt signs of sepsis found. Bordetella spp., Beta haemolytic Streptococcus, Coagulase-positive Staphylococcus, Coliform spp., and Pasteurella spp. were the most frequent bacterial species isolated in cases of sepsis. Oropharyngeal contamination was found in 308 submissions, and 315 proved nondiagnostic. There was no significant difference in age or sex between disease groups.

\section{STATEMENT (CONCLUSIONS)}

Bacterial infection of the lower respiratory tract is a frequent finding, more commonly made via cytological signs of sepsis, rather than microbial culture.

\section{Core outcomes in feline Chronic Kidney Disease: what should we be measuring?}

\section{Hannah Doit ${ }^{1}$, Marnie Brennan ${ }^{1}$, Rachel Dean², Marco Duz ${ }^{1}$, Richard Emes ${ }^{1}$}

1 University of Nottingham, Nottingham, United Kingdom

2 VetPartners, York, United Kingdom

\section{OBJECTIVES}

Over 100 different parameters are measured in the published treatment efficacy studies for cats with Chronic Kidney Disease (CKD), making it impossible to compare or combine the results of studies. Developing an agreed Core Outcome Set (COS) would resolve some of the issues as all future studies can use these when assessing treatment efficacy. The overall aim of this study was to create a COS for CKD in cats.

\section{METHODS}

Using a Delphi methodology, an anonymous, international panel of 73 stakeholders, including clinical experts, journal editors, regulatory agencies and cat owners, completed a series of online questionnaires to build consensus on the most important parameters to measure when treating cats with CKD. They were asked to rate the importance of parameters using Likert scales (1-9). Consensus was defined a priori as $80 \%$ of participants rating the parameter as 8 or 9 . The study contained 3 rounds in total.

\section{RESULTS}

Response rate was between 78 and 95\% across the first questionnaires, with 14 parameters reaching consensus for inclusion within the first round of the study. These included specific parameters from urine and serum tests, quality of life, survival time/renal survival end-point, IRIS stage and clinical examination.

\section{STATEMENT (CONCLUSIONS)}

This research highlights the outcomes which all stakeholders consider important when making treatment decisions for cats with CKD. Including this COS in future clinical trials will ensure results will be relevant to both first opinion and referral practice, strengthening the evidence base available for decision making.

\section{A cat and mouth game: Investigation of odontoclast dysregulation in feline tooth resorption}

\section{Nicola Mawson, Seungmee Lee, Professor Colin Farquharson, Dr Gurå Bergkvist}

The Royal (Dick) School of Veterinary Studies and the Roslin Institute, Edinburgh, United Kingdom

\section{OBJECTIVES}

Feline tooth resorption (TR) is a painful and progressive clinical condition that affects at least $30 \%$ of adult cats Recently, a RNA sequencing study (unpublished data) 